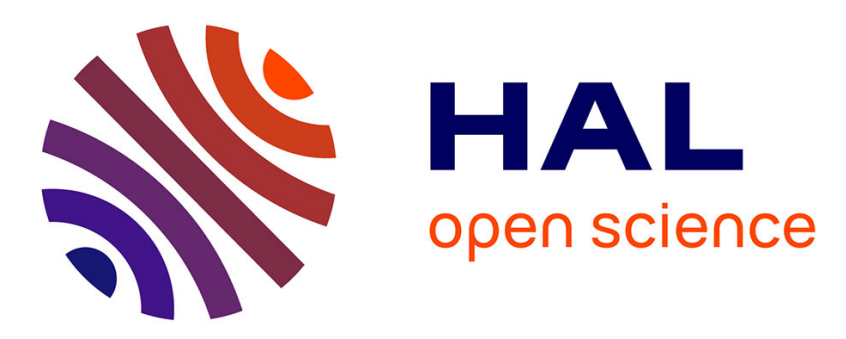

\title{
Resonant coupling between interface plasmons and surface plasmons at the junction between two simple metals
}

J. Kupersztych, Michèle Raynaud

\section{- To cite this version:}

J. Kupersztych, Michèle Raynaud. Resonant coupling between interface plasmons and surface plasmons at the junction between two simple metals. Solid Slate Communicalions, 1998, 106, pp.785 788. 10.1016/S0038-1098(98)00117-3 . hal-01949721

\author{
HAL Id: hal-01949721 \\ https://hal.science/hal-01949721
}

Submitted on 26 Dec 2020

HAL is a multi-disciplinary open access archive for the deposit and dissemination of scientific research documents, whether they are published or not. The documents may come from teaching and research institutions in France or abroad, or from public or private research centers.
L'archive ouverte pluridisciplinaire HAL, est destinée au dépôt et à la diffusion de documents scientifiques de niveau recherche, publiés ou non, émanant des établissements d'enseignement et de recherche français ou étrangers, des laboratoires publics ou privés. 
PII: S0038-1098(98)00117-3

\title{
RESONANT COUPLING BETWEEN INTERFACE PLASMONS AND SURFACE PLASMONS AT THE JUNCTION BETWEEN TWO SIMPLE METALS
}

\author{
J. Kupersztych* and M. Raynaud \\ Commissariat à l'Energie Atomique, DRECAM/SRSIM, Bât. 462, Centre d'Etudes de Saclay, \\ 91191 Gif-sur-Yvette Cedex, France
}

\begin{abstract}
(Received 6 February 1998; accepted 27 February 1998 by G. Bastard)
\end{abstract}

\begin{abstract}
The classic problem of the existence of genuine interface plasmons at the junction between two free-electron metals is revisited. By applying Bloch's standard hydrodynamical model for the homogeneous free-electron gas to the case of a bi-metal system, we demonstrate that localized interface plasmons resonantly coupled to surface plasmons do exist. It is shown that the amplitude of the resonant field at the metal-metal interface strongly depends on the layer thickness and disappears for a semi-infinite system. (C) 1998 Elsevier Science Ltd. All rights reserved
\end{abstract}

Keywords: A. metals, A. surfaces and interfaces, D. dielectric response, D. tunnelling.

The study of the dynamical dielectric response of coated metals has attracted renewed attention [1] because of its interest in topics like growth of oxide layers on metallic surfaces, optical properties of nonuniform metal films and electronic properties of metal-metal junctions. While these questions have been investigated for a rather long time [2], no definite answers have been given, even at the macroscopic level, to the conceptually simple problem of the collective electron behavior at the interface between two different metals.

The question of the existence of genuine interface plasmon modes was first considered by Stern and Ferrel [3] for the simplest model of a planar interface between two semi-infinite free-electron metals. In their description, the metals are characterized by (Drude) dielectric constants $\epsilon_{1}=1-\omega_{p 1}^{2} / \omega^{2}$ and $\epsilon_{2}=1-\omega_{p 2}^{2} / \omega^{2}$, where $\omega_{p 1}$ and $\omega_{p 2}$ denote their respective plasma frequencies. The corresponding plasmon fields thus represent collective electron oscillations of angular frequency $\omega=\left[\frac{1}{2}\left(\omega_{p 1}^{2}+\omega_{p 2}^{2}\right)\right]^{1 / 2}$, decaying exponentially on both sides of the interface. The Stern-Ferrel problem was generalized later on by Gadzuk [4] to waves propagating along the interface. In addition to the Stern-Ferrel modes that he recovered in the case of an overlayer of

\footnotetext{
* Corresponding author. E-mail: jkupersztych@cea.fr
}

large thickness, he gave a particular solution for the dispersion relation of the coupled modes taking dispersion effects into account and showed that, for a finite overlayer, the surface plasmon and interface plasmon may interfere.

However, the existence of the Stern-Ferrel modes in dispersive media still remains controversial. Several authors have re-examined the problem and claimed about the non-existence of these localized interface plasmons when dispersion is taken into account [5]. Besides, the influence of particular choices of boundary conditions [6] has been thoroughly examined and validity of the Bloch hydrodynamical model has been questioned [7].

In this paper, we shall reconsider the surface/ interface plasmon coupling problem initiated by Gadzuk [4] with a different approach. Thus, instead of assuming the form of a particular solution, we shall start from the basic equations, using again the hydrodynamical approach (which was the original theoretical framework within which the surface plasmon modes were discovered, indeed) and we shall deduce that localized interface plasmons do exist as the result of coupling with the field of the surface plasmons. We shall then see that the existence of this effect is actually strongly dependent on the layer thickness, so that these stimulated interface plasmons no longer exist for semi-infinite metal 
thickness, in agreement with some above-mentioned works [5].

In the standard widely-used hydrodynamical formalism, the collective oscillations of an electron gas are described by Poisson's equation:

$\boldsymbol{\nabla} \cdot \mathbf{E}(\mathbf{r}, t)=4 \pi \mathrm{e}\left[n_{0}-n_{e}(\mathbf{r}, t)\right]$,

the equation for conservation of matter:

$\partial n_{e}(\mathbf{r}, t) / \partial t+\nabla \cdot\left[n_{e}(\mathbf{r}, t) \mathbf{v}(\mathbf{r}, t)\right]=0$,

and the fluid equation of motion:

$\partial \mathbf{v}(\mathbf{r}, t) / \partial t+\frac{1}{2} \nabla v^{2}(\mathbf{r}, t)=-\mathrm{e} \mathbf{E}(\mathbf{r}, t) / m-\beta^{(\mathrm{i})^{2}} \nabla n_{e}^{(\mathrm{i})}(\mathbf{r}, t) / n_{0}$.

In these equations, $\mathbf{E}$ is the self-consistent electric field, $\mathbf{v}$ the fluid velocity, $n_{0}$ and $n_{e}$ denote, respectively, the ion and electron number densities and $\beta^{(i)}=\sqrt{\frac{3}{5}} v_{f}^{(i)}$ with $v_{f}^{(i)}=$ $\left(3 \pi^{2} n_{e}^{(i)}\right)^{1 / 3} \hbar / m$ is the Fermi velocity of metal (i). The last term in equation (3) represents the gradient of the kinetic pressure.

Let us consider the case of a two-layer planar metal system described within the framework of a jellium model (electron densities at equilibrium are neutralized everywhere by uniform background ion densities) consisting of a semi-infinite bulk (metal (I)) of electron number density $n_{e}^{(\mathrm{l})}$ overlaid by a foil (metal (II)) of electron number density $n_{e}^{(\mathrm{II})}$ and of thickness $h$ measured along the $z$-axis. In the present ultra-simplified description, we shall restrict ourself to investigate the linear response of the two coupled electron gas. Equations (1)(3) are linearized by writing $n_{e}^{(\mathrm{i})}(\mathbf{r}, t)=n_{e}^{(\mathrm{i})}+\tilde{n}^{(\mathrm{i})}(\mathbf{r}) \mathrm{e}^{-i \omega t}$ (with $\left|\tilde{n}^{(i)}\right| \ll n_{e}^{(i)}$, where $\mathrm{i}=\mathrm{I}$ and II in equations (1)-(3) which then take the following forms:

$\nabla \cdot \mathbf{E}(\mathbf{r})=-4 \pi \mathrm{e} \tilde{n}(\mathbf{r})$,

$-i \omega \tilde{n}(\mathbf{r})+\nabla \cdot\left[n_{e} \mathbf{v}(\mathbf{r})\right]=0$,

$i \omega n_{e} \mathbf{v}(\mathbf{r})=e_{e} \mathbf{E}(\mathbf{r}) / m-\beta^{2} \nabla \tilde{n}(\mathbf{r}) / n_{e}$.

Introducing the scalar potential $\Phi(\mathbf{r})$ of the electric field in equations (4) and (6) and by combining equations (4)(6), we obtain for describing the material medium the following familiar equation:

$\left[\Delta+\chi^{(i)^{2}}\right] \Delta \Phi^{(i)}(\mathbf{r})=0$

with $\chi^{(\mathrm{i})}=\left(\omega^{2}-\omega_{p}^{(\mathrm{i})^{2}}\right)^{1 / 2} / \beta^{(\mathrm{i})}$ where $\omega_{p}^{(\mathrm{i})}=\left(4 \pi \mathrm{e}^{2} n_{e}^{(\mathrm{i})} / m\right)^{1 / 2}$ is the plasma frequency of metal $(i)$ and, for vacuum:

$\Delta \Phi^{\mathrm{vac}}(\mathbf{r})=0$.

Now, we are looking for solutions of equations (7) and (8) in the form of waves propagating along the $(x, y)$-plane (say, along the $x$-axis) and exponentially decreasing along the $z$-axis at each interface (metal/metal and metal/vacuum). The general solution of equation (7) is a linear combination of solutions of homogeneous and inhomogeneous Laplace's equations [8]. Taking into account the condition of no divergence of the potential $\Phi^{(\mathrm{I})}$ for $z=-\infty$ and the transitional invariance of the potentials along the $x$-axis, it can be written for each medium (i) as:

$\Phi^{(\mathrm{I})}(\mathbf{r})=\left[A_{\mathrm{I}} \mathrm{e}^{i k x}+A_{\mathrm{I}}^{\prime} \mathrm{e}^{-i k x}\right]\left[B_{\mathrm{I}} \mathrm{e}^{k z}+C_{\mathrm{I}} \mathrm{e}^{\zeta^{(\mathrm{II}} z}\right]$

and

$$
\begin{aligned}
\Phi^{(I I)}(\mathbf{r})= & {\left[A_{\mathrm{II}} \mathrm{e}^{i k x}+A_{\mathrm{II}}^{\prime} \mathrm{e}^{-i k x}\right]\left[B_{\mathrm{II}} \mathrm{e}^{k z}+B_{\mathrm{II}}^{\prime} \mathrm{e}^{-k z}\right.} \\
& \left.+C_{\mathrm{II}} \mathrm{e}^{\zeta^{(1)} z}+C_{\text {II }}^{\prime} \mathrm{e}^{-\zeta^{(I I)} z}\right],
\end{aligned}
$$

where $\zeta^{(\mathrm{i}) 2}=k^{2}-\chi^{(\mathrm{i}) 2}$. The solution of Laplace's homogeneous equation (8), taking into account the condition of no divergence of the potential $\Phi^{\text {vac }}$ for $z=+\infty$, has the form:

$\Phi^{\mathrm{vac}}(\mathbf{r})=\left[A_{\mathrm{vac}} \mathrm{e}^{i k x}+A_{\mathrm{vac}}^{\prime} \mathrm{e}^{-i k x}\right] B_{\mathrm{vac}} \mathrm{e}^{-k z}$.

These expressions differ generally from Gadzuk's particular solution [4] in which he explicitly assumed $k=\zeta^{(\mathrm{I})}=\zeta^{(\mathrm{II})}$. In the above expressions the constant coefficients are, of course, determined by means of the boundary conditions. To determine these conditions, we bring into play the fact that we are looking for an interaction between the two metals in the form of a surface plasmon tunnelling through the metal-metal interface. [It is tacitly assumed that the surface plasmon is created by any external source of energy (laser or electron beam), which therefore means that our physical system is an open system. Hence, conservation of the energy current density may not be imposed.J. For this phenomenon to occur, the self-consistent electric field $\mathbf{E}$, within the framework of the present plasma-type description of the two metals, has to be continuous throughout the metal-metal interface. The usual continuity condition of the electric displacement [which writes here $\mathbf{D}=\mathbf{E}+(4 \pi / i \omega) \mathbf{j}]$ then gives, as a consequence, that the electron current density $\mathbf{j}$ must also be continuous at the metal-metal interface. Furthermore, the familiar boundary conditions at the metal-vacuum interface are: continuity of the normal and tangential components of the electric field $\mathbf{E}$ together with continuity of the normal component $j_{z}$ of the electron current density. Hence, the desired expressions of the dispersion relation $\omega(k)=0$ and of the components of the electric field are easily obtained after a straightforward calculation. Explicitly, the dispersion relation, which is nothing else but the determinantal equation of the 
linear equation system between the above coefficients, is:

$$
\begin{aligned}
& \left(\zeta^{1}-k\right)\left[1-\left(\omega_{p}^{\mathrm{II}}-\omega^{2}\right) /\left(\omega_{p}^{\mathrm{I}^{2}}-\omega^{2}\right)\right] \mathrm{e}^{-\left(k+\zeta^{\mathrm{II}}\right) h} \\
& +\left(\zeta^{\mathrm{I}}-\zeta^{\mathrm{II}}\right) S_{-} \mathrm{e}^{-2 \zeta^{\prime \prime} h}+\left(\zeta^{\mathrm{l}}+\zeta^{\mathrm{II}}\right) S_{+}=0,
\end{aligned}
$$

where

$S_{ \pm}=\frac{1}{2}\left(1 \pm k / \zeta^{I I}\right)\left(\omega_{p}^{\mathrm{II}} / \omega\right)^{2}-1$,

and the normal components of the electric field in the three regions I, II and vacuum, are:

$$
\begin{aligned}
E_{z \leq-h}^{\mathrm{I}}= & -F(x) S_{-}^{-1}\left\{\zeta^{\mathrm{I}} G \mathrm{e}^{\zeta^{\mathrm{I}}(z+h)}\right. \\
& \left.+k \mathrm{e}^{k(z+h)}\left(\omega_{p}^{\mathrm{II}}-\omega^{2}\right) /\left(\omega_{p}^{\mathrm{I}^{2}}-\omega^{2}\right)\right\} \mathrm{e}^{-k h}
\end{aligned}
$$

$E_{-h \leq z \leq 0}^{\mathrm{II}}=-F(x) S_{-}^{-1}\left\{\zeta^{\mathrm{Il}} S_{-} \mathrm{e}^{\zeta^{\prime \prime} z}-\zeta^{\mathrm{II}} S_{+} \mathrm{e}^{-\zeta^{\prime \prime} z}+k \mathrm{e}^{k z}\right\}$

and

$E_{z \geq 0}^{\mathrm{vac}}=F(x) S_{-}^{-1} k\left[\left(\omega_{p}^{\mathrm{II}}-\omega^{2}\right) / \omega^{2}\right] \mathrm{e}^{-k z}$,

where

$$
F(x)=\left[A_{11} \mathrm{e}^{i k x}+A_{11}^{\prime} \mathrm{e}^{-i k x}\right]
$$

and

$$
\begin{aligned}
G= & S_{-} \mathrm{e}^{\left(k-\zeta^{\prime \prime}\right) h}+S_{+} \mathrm{e}^{\left(k+\zeta^{\prime \prime}\right) h} \\
& +1-\left(\omega_{p}^{I^{2}}-\omega^{2}\right) /\left(\omega_{p}^{\mathrm{I}^{2}}-\omega^{2}\right) .
\end{aligned}
$$

At this stage, it is worthwhile to examine the behavior of these expressions in different limit cases.

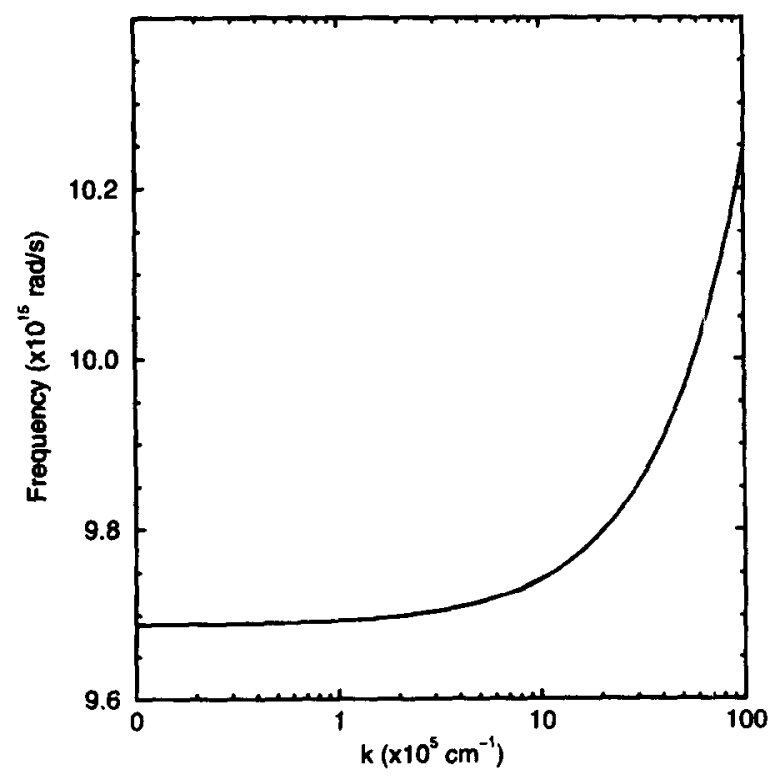

Fig. 1. Representation of the dispersion relation $\omega(k)=0$ given by equation (9).
For example, when the layer thickness $h \rightarrow 0$, equation (9) gives

$\frac{1}{2}\left(1+k / \xi^{l}\right)\left(\omega_{p}^{1} / \omega\right)^{2}-1=0$,

which is nothing other than the dispersion relation of surface plasmons [9] corresponding to metal $M(\mathrm{I})$. Besides, the same is true when the electron number densities of the two metals $n_{e}^{(\text {I) }}$ and $n_{e}^{(\text {II) }}$ are equal. It is also worth noting that, in the case of a semi-infinite layer of metal $M$ (II) (which is obtained for $h \rightarrow \infty$ ), this relation reduces simply to:

$S_{+} \equiv \frac{1}{2}\left(1+k / \zeta^{\mathrm{II}}\right)\left(\omega_{p}^{\mathrm{II}} / \omega\right)^{2}-1=0$,

which is again the dispersion relation [9] of surface plasmons corresponding to metal $M$ (II). This remark yields the following important consequence: as the layer thickness increases, the solution $\omega(k)$ of the dispersion relation (9) tends towards the surface plasmon frequency of the metal layer [that is, $\omega_{p}^{\mathrm{II}} / \sqrt{2}$ (for $k=0$ )]. In other words, for large layer thickness, the self-consistent field becomes independent of the substratum.

The sought-after effect can be perceived analytically on the equations (11)-(13), owing to the exponential behavior of the field components in the three regions: metal (I), metal (II) and vacuum. Thus, the expression (13) shows that the field in vacuum presents a sharp maximum at the surface $(z=0)$, which is nothing other than the surface plasmon. Next, the expression (11) also shows that the self-consistent electric field presents another sharp maximum for $z=-h$, that is, at the metal (I)/metal (II) interface. This is just the soughtafter interface plasmon coupled to the field of the surface plasmon. Furthermore, the role of the layer thickness is revealed by the factor $\mathrm{e}^{-k h}$ in eqaution (11) and shows a decreasing of the resonance amplitude as the thickness increases. This behavior admits an obvious explanation: when $h$ is large, the surface plasmon field must tunnel through too large a distance to enhance the charge fluctuations at the metal-metal interface and the interface plasmon decreases correspondingly. Hence, the stimulated interface plasmon disappears for large layer thickness, in agreement with previous studies [5].

For exploring numerically the above results, we have considered as possible example of a bi-metal system $(M(\mathrm{I}) / M(\mathrm{II}))$ the system (Al/Au). On Figs 2 and 3 are reported (in arbitrary units) the amplitude variations of the normal component of the self-consistent electric field $E_{z}(\omega, k)$ with respect to the $z$-coordinate, where the frequency $\omega$ and wave-vector $k$ are, of course, for each layer thickness $h$, given by the solution of the dispersion relation (9) represented on Fig. 1. The above-mentioned effect appears strikingly: the self-consistent electric 


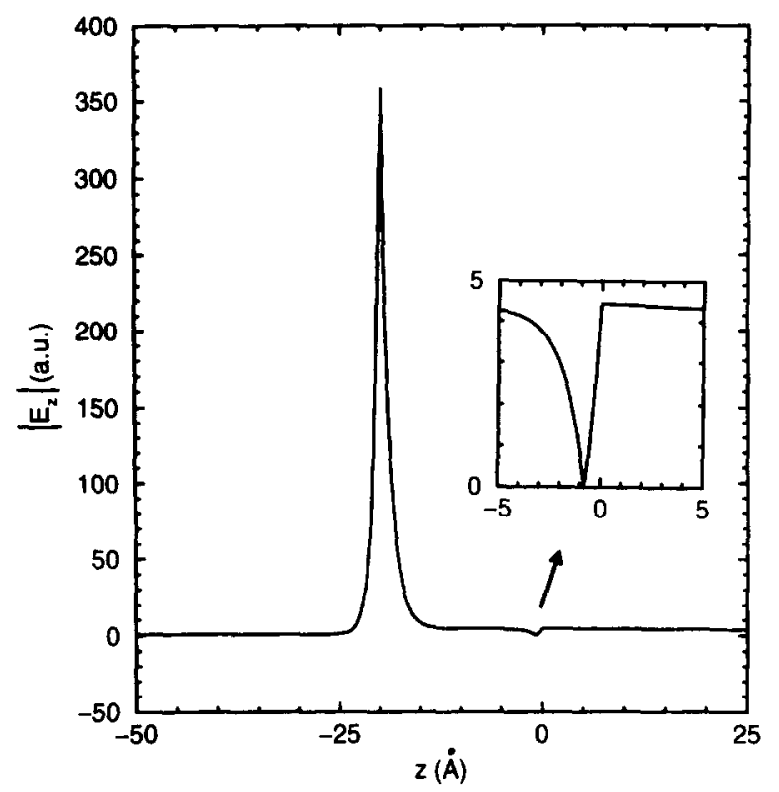

Fig. 2. Representation of the normal component of the self-consistent electric field $E_{z}(\omega, k)$ (at $t=0$ ) for the system Al/Au $\left(\omega_{p}^{(\mathrm{Al})}=2.41 \times 10^{16} \mathrm{rad} \mathrm{s}^{-1}, \omega_{p}^{(\mathrm{Au})}=\right.$ $\left.1.37 \times 10^{16} \mathrm{rad} \mathrm{s}^{-1}\right)$, vs normal coordinate $z$. We have taken $k=5 \times 10^{5} \mathrm{~cm}^{-1}$. The thickness of the Au-layer is $h=20 \AA$.

field exhibits a strong enhancement at the metal-metal interface (at $z=-h$ ). This stimulated interface-plasmon field is thus characterized by an amplitude and a spatial extension much greater and narrower than that of

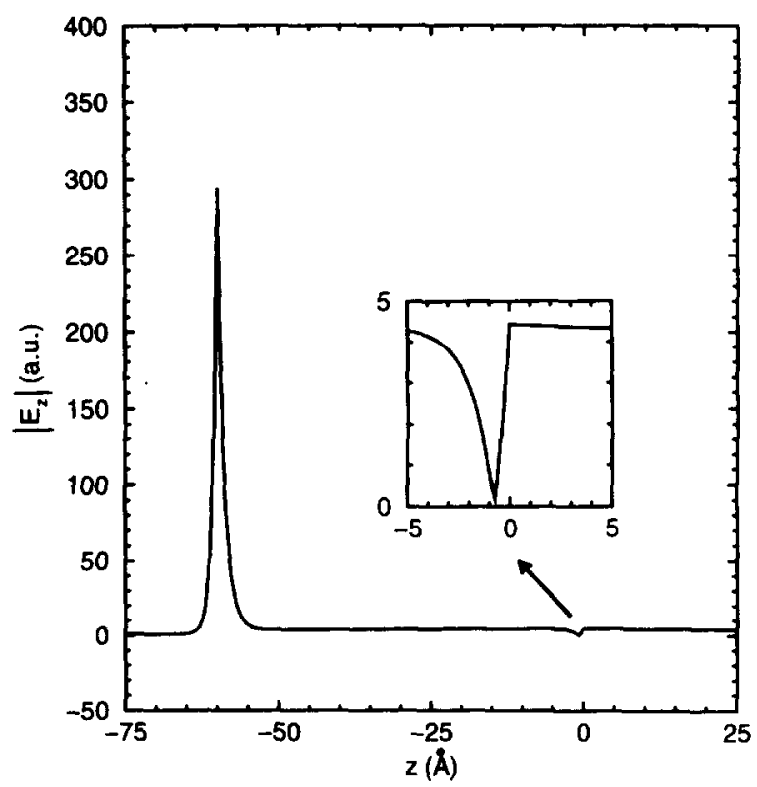

Fig. 3. Same caption as for Fig. 2 but here $h=60 \AA$. the surface plasmon (at $z=0$ ). The decreasing of the amplitude of the interface plasmon field when the layer thickness $h$ increases is also clearly exhibited.

In conclusion, we have analytically demonstrated that interface plasmons resulting from the coupling with the field of the surface plasmon do exist, this effect depending strongly on the thickness of the overlayer metal. However, as our present purpose was focussed onto the existence of the effect in question, the hypothesis and the approximations inherent of the model have been simplified at maximum, which makes any quantitative prediction for real metal systems rather difficult. This is, of course, the case of very thin overlayers (a few Angströms) since the macroscopic (hydrodynamic) description is not applicable. Other important questions are the role played by interband transitions that are expected to restrain the surface/ interface plasmon coupling, the influence of retardation effects that are to be taken into account for small wavevectors (of the order of or smaller than $\omega_{p} / c$ ) and the effect of electron density structure at the metal surfaces on plasmon dispersion. We hope to study these questions in a future paper.

\section{REFERENCES}

1. Gaspar, J.A. et al., Phys. Rev. Lett., 67, 1991, 2854; Albuquerque, E.L. and Cottam, M.G., Physics Reports, 233, 1993, 67; Zharnikov, M., Mehl, D. and Steinruck, H.P., J. of Elec. Spect., 70, 1994, 103.

2. Inglesfield, J.E. and Wikborg, E., J. Phys. F: Metal Phys., 5, 1975, 1706. For a detailed review see e.g. Boardman, A.D., Electromagnetic Surface Modes (Edited by A.D. Boardman). Wiley, New York, 1982 with numerous references herein.

3. Stern, E.A. and Ferrell, R.A., Phys. Rev., 120, 1960 , 130.

4. Gadzuk, J.W., Phys. Rev., B1, 1970, 1267.

5. Summerside, P., J. Phys. F: Metal Phys., 9, 1979, 1643; Uberoi, C. and Reyes, J., Pramana, 18, 1982, 237; Pimpale, A. and Paranjape, B.V., Surf. Sci., $161,1985,69$.

6. Boardman, A.D., Surf. Sci., 112, 1981, 153; Jewsbury, P., J. Phys. F: Metal Phys., 11, 1981, 195.

7. Kleinman, L., Phys. Rev., B7, 1973, 2288; Ahlquist, P. and Apell, P., Physica Scripta, 25, 1982, 587; Schwartz, C. and Schaich, W.L., Phys. Rev., B26, 1982, 7008.

8. Courant, R. and Hilbert, D., Methods of Mathematical Physics, Vol. I. Wiley, New York, 1989.

9. Ritchie, R.H., Phys. Rev., 106, 1957, 874; Ritchie, R.H., Prog. Theor. Phys., 29, 1963, 607. 